\title{
Morbilidad y estado nutricional en lactantes de alto riesgo. Estudio de terreno
}

\author{
Sylvia Cruchet M. ${ }^{1}$; Magdalena Araya Q.1; \\ Julio Espinoza M.'; Oscar Brunser T. ${ }^{1}$
}

\section{Morbidity and nutritional status in children of high risk of morbidity. A field study}

\begin{abstract}
A predictive model which identifies intants with four or five limes higher morbidily rales than unselected populalion, wos used to evaluate 108 intanls; 41 of whom lost $10 \%$ or more of their weight/age ratio along a six monlh follow up. Frecuencies and lype of diseases did nol allow to differenciale patients whose nutrition deteriorated from hose in which it did not. The greatest decreases of weigh lor age were observed in the second six months of lile. In $77.1 \%$ of children weighl goin improved ofter 12 months of age, when energelic requirements decrease. Results suggesl that the risk: for heolth of those childien may be rather associaled with maternol inappropriate practices than lo biological or environmental voriables.
\end{abstract}

[Key words: risk factors, infont nutrition, molnutrition, morbidily.]

En los últimos affos se ha puesto énfasis en la necesidad de identificar grupos que tienen alto riesgo de sufrir determinadas enfermedades, de manera de desarrollar programas preventivos específicos $^{1-4}$. En este contexto hemos realizado estudios sobre enfermedad diarreica utilizando un modelo predictivo que identifica niños que sufren 4 a 5 veces más episodios de morbilidad que los no seleccionados 5,6 . En estos lactantes se ha observado, además, que su estado nutricional se deteriora significativamente en los 6 meses de observación que duran los seguimientos realizados $^{5,7} ; 29 \%$ de los nifos perdio más de $10 \%$ de su relación peso/edad inicial, mientras que en algunos la pérdida llegó al 20\%; en varias oportunidades se formularon diagnósticos precisos, como por ejemplo enfermedad celíaca, síndrome bronquial obstructivo crónico, anemia ferropriva, síndromes convulsivos y otros. Como para efectuar estos diagnósticos se requieren estudios especializados, $y$, por otra parte, en estas mismas cohortes habíamos detectado que las madres de estos niños concurren escasamente a los servicios de salud, se planificó este estudio para eva-

1. Unidad de Gastscenterología del Instituto de Nutrición y Tecnología de los Alimentos.

Fuente de financiamiento: DTI ProyecLo M3017/921F. luar, en un grupo de niños seleccionados por mcdio del predictor de riesgo, el tipo de afección, la oportunidad de su diagnóstico y su relación con el estado nutricional.

\section{Material y Método}

El estudio se realizó entre julio dc 1990 y junio de 1991, identificándose a todas las familias que tuvieran un nino menor de un año inscrito en el Consultorio La Faena, en cl átea Oriente de Santiago $(n=1100$ ). Se pudo contactar en el domicilio registrado en la ficha clínica a 678 de ellas, cuyos hijos tenían entre 6 y 12 meses de edad. En 20,4\% $(n=138)$ el predictor fue positivo y se inició su seguimiento, divididos en dos cohortes consecutivas: 59 de los que formaron la primera cohorte (julio a diciembre) y 49 de los de la segunda (encro a junio) terminaron el seguimiento. La deserción de 30 niños se debió a cambio de domicjlio de las familias o bien a que la madre empezó a trabajar, llevando al hijo consigo. Este diseño permitió obtener datos durante

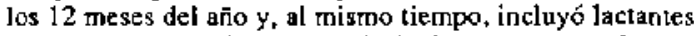
menores tanto en los meses de inviemo como en los de verano. Las familias fueron visitadas en sus domicilios semanalmente, o con la frecuencia que fuera necesaria, por una enfermera de terreno, quien registró la presencia de síntomas en el nif́o índice: además, cuando éste estaba enfermo motivó a las madres a consultat a nuestro equipo en una esısción de campo, localizada en el Consultorio La Faena. En este lugar se trataron las enfemedades y se efectuó mensualmente un control de niño sano. De los 108 niños que terminaton el seguimiento, 41 perdiemon más de $10 \%$ de su relación peso/edad inicial, calculada en base a las tablas 
OMS/NCHSs. A todos ellos, al final del periodo de seguimiento, se les investigaron nuevamente los antecedentes clínicos, se les practicó un examen físjico completo, hemograma, sedimento de orina $y$ urocultivo, examen coproparasitario seriado (3 muestras) 9,10 y cualquier otro que se considerara clínicamente pertinente, con el objeto de hacer el diagnóstico y de entender la causa de su deterioro nu. tricional.

\section{Resultados}

De los 41 niños seleccionados e invitados a asistir a la estación de campo para evaluación médica sólo respondieron las madres de 35 (20 de la primera cohorte y 15 de la segunda); pese a múltiples citaciones (hasta 10 veces), las restantes nunca llegaron a consultar $(6 / 41=14,6 \%)$. Entre los 35 lactantes evaluados se diagnosticó un caso de alergia a proteína de leche de vaca y dos de sindrome bronquial obstructivo recidivante $(3 / 35=8,6 \%)$. Los otros diagnósticos efectuados correspondieron a episodios infecciosos "banales" a repetición, tales como infecciones respiratorias altas (87 episodios), diarrea aguda (51 episodios), bronquitis aguda (92 episodios), otitis aguda (11 episodios), conjunlivitis aguda ( 5 cpisodios), enfermedades infectocontagiosas (8 casos) y dermatitis (11 casos). Los exámenes de laboratorio permitieron detectar 3 casos de anemia ferropriva y 5 enteroparasitosis ( 3 giardiasis y 2 amebiasis). Tres niños no concurrieron a la toma de los exámenes solicitados a pesar de las facilidades dadas (dinero para el transporte o la oferta de la enfermera de llevar personalmente al niño). Los tratamientos medicamentosos se efectuaron sin problemas, ya que se contaba con recursos adicionales para este propósito.

El número de episodios mórbidos y su duración promedio en los niños que se detcrioró y en los que no se deterior 6 su estado nutricional, aparece en la tabla. Desde el punto de vista clínico no se detectaron diferencias en la calidad de los síntomas encontrados en los dos grupos.

Se encontró una relación peso/edad por debajo del $90 \%$ del percentil 50 de las tablas OMS/ NCHS en 12/41 (29,3\%) de los niños al inicio del seguimiento, en $23 / 41(56,1 \%)$ durante algún período del seguimiento y en $14 / 41(34,2 \%)$ al final de éste. En el análisis de la evolución nutricional se obtuvieron dos tipos de curvas: los lactantes cuyo estado nutritivo se deterioró y no se recuperó durante el período de observación ( 8 casos) (figura 1); y aquellos cuyo cstado nutricional se deterioró inicialmente y a partir de los 13 a 15 meses de edad comenzaron a mejorar progresivamente (27 casos) (figura 2).

\section{Comentario}

Como en evaluaciones anteriores, alrededor de $20 \%$ de las familias de nivel socioeconómico bajo atendidas en La Faena tuvieron predictor positivo ${ }^{5,11}$. De los nifnos así seleccionados, $38 \%$ (4]/108) perdieron más del $10 \%$ de su relación peso/cdad inicial en los 6 meses de seguimiento. La proporción de desnutridos dentro del grupo de 108 niños de riesgo es mucho mayor $-11,1 \%$ al inicio, $21,3 \%$ en algún momento del seguimiento y $13,0 \%$ al final- que en la población general del país de edad comparable ${ }^{12,13}$.

Esto representa un "bolsón" de niños desnntridos que merecerían atención especial por parte de los servicios de salud. Aquellos que mejoraron su ganancia ponderal $(n=27,77,1 \%)$, lo hicieron a partir de los 13 meses de edad. El mayor deterioro se produce alrededor de la milad del segundo semestre de la vida, cuando los requerimientos energéticos comienzan a elevarse, $y$ disminuye al final del primer año cuando dichos requerimientos comienzan a descender nuevamente ${ }^{14.15}$. Estos resultados sugicren que la mejoría observada estaría asaciada al aumento de edad y no a la disminución del riesgo. Desde otro punto de vista, estos resultados también sugieren que los requerimientos del infante no son cubiertos durante el segundo semestre de vida $y$, por lo tanto, el énfasis de los programas de salud

Tabla

\begin{tabular}{lrr}
\hline & $\begin{array}{c}\text { Grupo con } \\
\text { deterioro } \\
\text { nutricional }\end{array}$ & $\begin{array}{c}\text { Grupo sin } \\
\text { deterioro } \\
\text { nutriclonal }\end{array}$ \\
\hline $\begin{array}{l}\text { Díasiniños } \\
\text { observados } \\
\text { Total de episodios }\end{array}$ & 6388 & 11315 \\
$\begin{array}{l}\text { Episodios/ } \\
\text { niño/año }\end{array}$ & 272 & 467 \\
$\begin{array}{l}\text { Duración promedio } \\
\text { de los episodios (días) }\end{array}$ & 11,7 & 15,5 \\
\hline
\end{tabular}




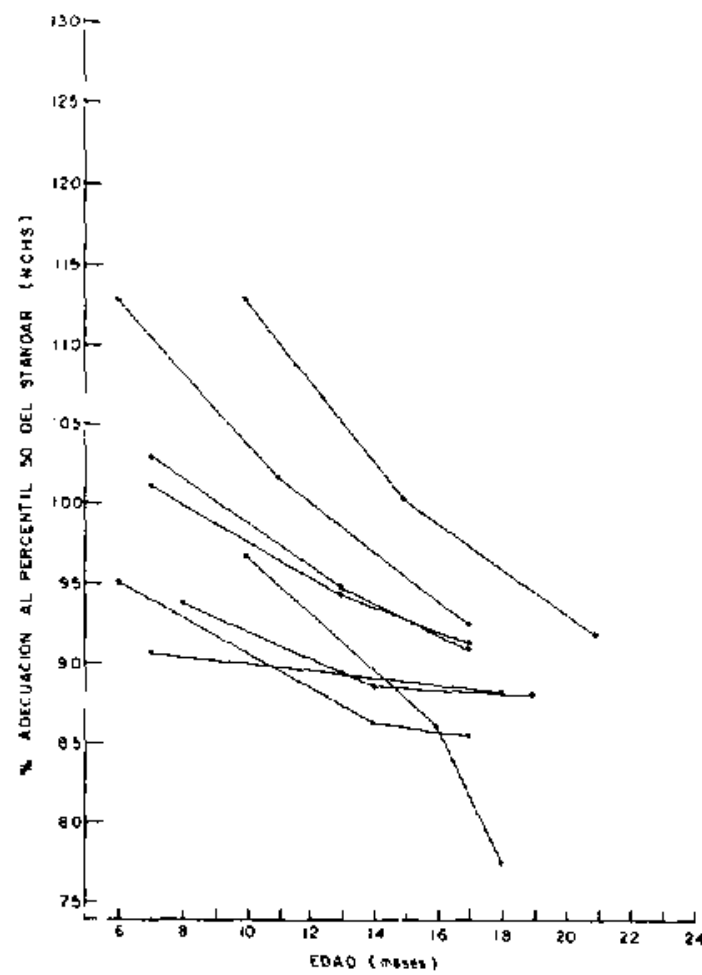

Figura 1: Evvolución de la relación peso/edad en lactantes de riesgo que no recuperaron este parámetro durante el seguimiento.

que atienden cstas necesidades debieran tomar en consideración que una proporción de los ninos no reciben el beneficio del programa porque sus madres se marginan de ellos. Pará incorporarlos a dichos programas sería necesario buscarlos dirigidamente y utilizar métodos distintos a los habituales para identificarlos y lograr que las madres participen en ellos.

Llama la atención que $14,6 \%$ de los lactantes, cuyo estado nutritivo se detcrioró, no recibicron el beneficio de la atención pediátrica preferencial que les ofrecimos porque no se logró que sus madres asistieran a la estación de campo, lo que revela una actitud muy especial en ellas frente al cuidado de sus hijos ${ }^{\text {t6. }}$.

La mayoría de los niños presentó sólo episodios mórbidos "banales", del tipo descrito por Mata en niños guatemaltecos de nivel socioeconómico bajo ${ }^{17}$. Este autor postuló que et deterioro nutricional se debcría a la repetición de los episodios. Sin embargo, en nuestro estudio, no se

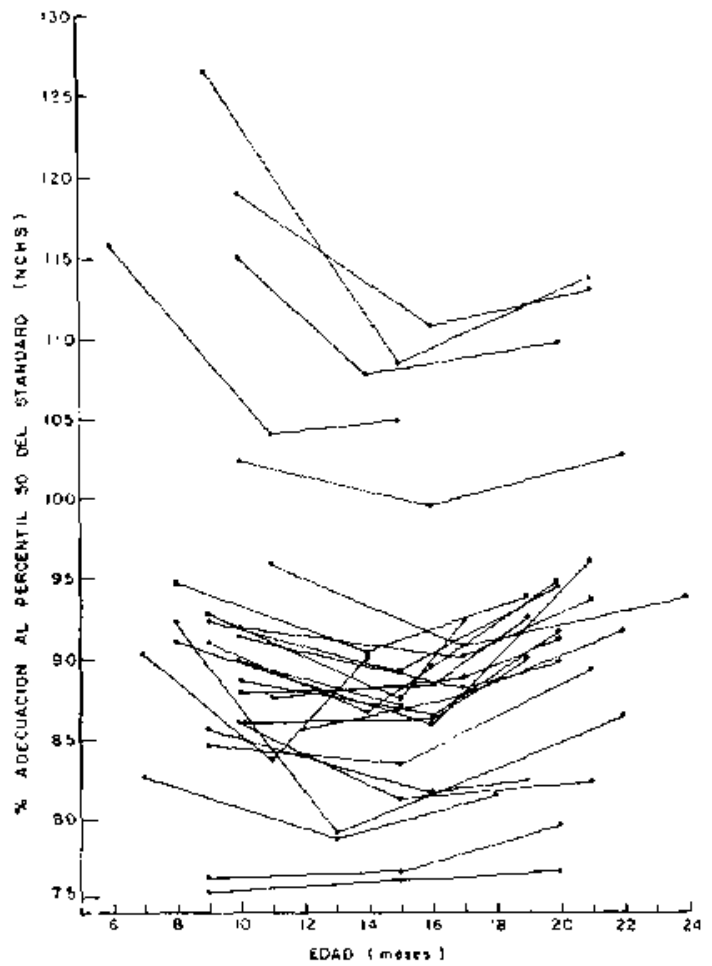

Figura 2: Evolución de la relación peso/edad en jactantes de riesgo que recupcraron este parámetro durante el seguimiento.

encontraron difercncias significativas en el tipo de afecciones detectadas ni en su duración, entre los niffos en quienes se deterioró o no el estado nutricional. Las enfermedades más graves, que fueron parte importante de la motivación para realizar este estudio, se pesquisaron en sólo 3 de los 35 casos $(8,6 \%)$. Si se toma en consideración que el número, la calidad y la duración de las afecciones fueron comparables en ambos grupos, parece razonable postular que el factor que favorece el deterioro nutricional no estaría relacionado a las enfermedades que sufre el niño sino a otros factores, entre los que deben incluirse las prácticas inadecuadas de la madre ${ }^{18,19}$.

\section{Resumen}

La identificación de grupos de personas con mayor riesgo de enfermar permite focalizar las acciones de salud con mayor eficiencia. Utilizan- 
de un modelo predictivo que selecciona lactantes que sufren 4 a 5 veces más morbilidad que la población general, se evaluaron 108 lactantes, de los cuales 4l sufrieron deterioro superior a $10 \%$ de la relación peso/edad durante un seguimiento de 6 mises. La frecuencia y tipo de enfermedades no diferenció a los grupos con y sin deterioro de la nutrición. El mayor daño nutricional se observó durante el segundo semestre de vida. $E_{n} 77,1 \%$ de los niños mcjoró su ganancia ponderal después del año de vida, lo que coincide con la disminución de los requerimientos energéticos. Los resultados sugieren que las conductas y prácticas maternas y no las variables biologicas o ambientales podrían explicar el mayor riesgo de los niños.

(Palabras clave: factores de riesgo, nutrición de lactantes, desnutrición, morbilidad.)

\section{Agradecimientos}

Al Dr. J. Pino, M.C., director del Consultorio La Faena, y a su personal, por las facilidades prestadas para la realización de este proyecto. A la Sra. Elvira Fontecilla, por su cxcelente labor en la recolección de la información de terreno.

\section{Referencias}

1. Stamier $D$, Wentforth $D$, Neaton $D$ : Is relationship bctween serum cholesterol and risk of premature dealh from coronary heart disease continuous and graded? Findings in 356222 primary screenees of the multiple risk factor intervention Trial (MRFTT). JAMA 1986; 256: 2823-2828.

2. Steering Committee of the Physicians' Health Study Research Group. Final report on the aspirin component on the ongoing Ihysicians "Health Sudy. N Fing J Med 1989; 321: 129-135.

3. Davies B, Peiros-Barvasian A: El concepto de riesgo en la asistencia sanitaria. OMS. Cuadernos de Salud Pública $\mathrm{N}^{2} 76$. Organización Mundial de la Salud. Ginebra, 1985.

4. Monckeberg F, Mardones-Restat F Valiente S: The evolution of malnutrition and monality in infants and young children in the last 20 years in Chile. En: New developments in the analysis of mortality and causes of death. Hansluwka II, Lópcz AD, Porapkkham Y, Prasartkul P, eds. Bangkok: Mahidol University. 1986. 295-321.
5. Araya M, Baiocchi N, Espinoza J, Brunser O: Persislent diarthoea in the community. Characteristics and risk factors. Acta Paediatr Scand 1991; 80: 181-189.

6. Araya $M$, Espinoza J, Brunser $O$, Pacheco l, Cruchet $S$. Aplicación de un modelo predictivo en niños de nivel sociocconómico bajo. Rev Med Chil 1992; 120: 342-348.

7. Espinoza J, Araye $M$, Pocheco I, Brunser O, Crucher $S$ : Evolución del estado nutricional de lactantes elegidos con un predictor de riesgo. XXVII Congreso de la Sociedad Latinoamericana de Investigación Pediátrica. San Pablo, Brasil. Resumen 33. 1990.

8. Hamill PV, Drizd TA, Johnson CH, et al.: Physical growth: National Health Centcr for Health Siatistics percentiles. Am J Clin Nutr 1979; 32: 607-629.

9. Burrows RBA: A new fixative tcchnique for the diagnosis of intestinal parasites. Am J Clin Pathol 1967; $48: 342-346$.

10. Sagua II: Comparación del rendimiento de tres mctodos en el diagnóstico coproparasitológico. Rev Med Chil 1975; 103: 175-177.

11. Araye M. Espinozo S, Briwser O. Cructet S, Pacheco I: Prevcnción de morbilidad en lactantes scleccionados por un predictor de riesgo. XXVIII Reunión snual de la Sociedad Latinoamericana de Investigación Pediátrica, San Pablo. Brasil, 1990; Resumen N 34.

12. Instaturo Nacional de Estadísticas: Demografía Año. 1989. Santiago: lnstituto Nacional de Estadísticas: Departamento de Impresiones y Distribución. 1990.

13. Aibala C. Alvarez ML, Agunyo $M$, es al.: Prevención de la desnutrición durante los primeros años de vida. En: Desnutrición isfantil. F Monckeberg, cd, Santiago: Impresora Creccs. 198B: 139-171.

14. Whisehead RG. Paul AA: Human lactation, infant feeding and growth: secular trends. En: $M$ Gracey y $F$ Falkner, eds. Nutritional neds and assessment of normal growth. Vevey/Nestlé Nuirivion. New York: Raven Press. 1985: 85-122.

15. Mata LJ: Environmental factors affecting nutrition and growth. En: M Gracey y F Falkner, eds. Nutritional need 5 and assessment of notmal growth. Vevey/Neslé Nutrition. New York: Raven Press. 1985: 165-184.

16. Mala $W$; 'The children of Sanla Maria Cauqué: a prospcctive field study of health and growth. Cambridge: MIT Press, 1978.

17. Mala $L$, Urruta $J J$, Albertazzi $C$, Pellecer $O$. Arellano $E$ : Influence of recursent infections on nutrition and growth of children in Guatemala. $\mathrm{Am} \mathrm{J}$ Clin Nutr $1972 ; 25 ; 126 \%-1275$.

18. Humphreys $D$, Araya $M$, Espinoza $J$, Roizblan $A$, Brutser $O$, Pacheco I: Evaluación de la salud mental de madres de niños de alto rícsgo. XXIX Reunión Anual de la Sociedad Latinoamericana de Investigación Pediálica. Viña del Mar. 1991. Resumen 24.

19. Zegers B. Fernández CL, Araya M: Caraclenísticas de personalidad de madres de nivos con alto riesgo de cofermar. XXIX Reunión Anual de la Sociedad latinoameticana de Investigación Pediátrica. Viña del Mar. 1991. Resumen 35. 